\title{
Biomechanical assessment and finite element analysis of a simulated axial-loading experiment on a wrist protector model
}

Jian Ying He

Jiangxi Provincial People's Hospital

Li Zhang ( $\sim$ hejianying626@163.com )

Jiangxi Provincial People's Hospital

Research article

Keywords: Wrist, biomechanical assessment, finite element analysis, wrist protector

Posted Date: March 26th, 2020

DOI: https://doi.org/10.21203/rs.3.rs-17828/v1

License: (c) (1) This work is licensed under a Creative Commons Attribution 4.0 International License.

Read Full License 


\section{Abstract}

Objective To evaluate the biomechanical analysis and effect of the wrist protector and provide a theoretical basis for wrist fractures and the optimal design of wrist protectors. Methods 6 cadaveric wrist models were axially loaded $600 \mathrm{~N}$ stress, and the stress magnitude and distribution of the experimental group (wearing wrist protectors) and control group were obtained. Furthermore, a three-dimensional finite element analysis was conducted to verify the scientificity and effectiveness of the models. Results The stresses on the radial distal palmar, ulnar distal palmar, radial distal dorsal, ulnar distal dorsal, radial proximal palmar and ulnar proximal palmar units in the experimental group were lower than those in the control group $(P<0.05)$. However, the stresses on the radial proximal dorsal and ulnar proximal dorsal units were higher than those in the control group $(P>0.05)$. Conclusion The stress on the radioulnar palmar unit was high, while the radioulnar dorsal unit one was relatively low. Within the range of physiological loads, wearing wrist protectors can significantly reduce the stress on the radioulnar distal palmar, radioulnar proximal palmar and radioulnar distal dorsal units.

\section{Introduction}

The incidence of wrist fracture is currently widespread, accounting for $6.7-11 \%$ of all fractures in the body, which inevitably results in high medical costs $[1,2]$. The wrist joint is mainly composed of a bony structure and other small joints and is a composite joint between the forearm and palm [3, 4]. Moreover, not only do the bony structure and other small joints have a clear division of labor in structure but these components also correspond to each other in function [5,6]. As the most complicated joint in the human body [7], the mechanical mechanism of the wrist joint is complex. Once the wrist joint is injured, it is likely

to cause secondary damage to other wrist structures [8], affecting the function of the relevant parts of the upper limbs. Therefore, a thorough and detailed study of wrist joints has important clinical significance.

At present, studies on the prevention of wrist fracture are still limited to drugs in the treatment of osteoporosis, osteoporosis fracture predicted by QCT, etc. [9-12]. However, from the perspective of biomechanics and finite element analysis, it is of great practical significance to explore the prevention and treatment of wrist fractures. Therefore, this paper combines a mechanical experiment on a cadaveric wrist model with finite element wrist simulation analysis and verifies each, which contributes to the biomechanical analysis and effect evaluation of wrist protectors. Furthermore, this research provides a theoretical basis for simulation experiments on wrist injuries and the optimal design of wrist protectors.

\section{Materials And Methods}

Materials

Biomechanical testing. Wet wrist samples $(n=6)$ were collected from cadavers (age at mortality of 20-50 years) from the Department of Human Anatomy of Basic Medicine, Nanchang University (Nanchang, China). Prior to storage at $-20^{\circ} \mathrm{C}, \mathrm{X}$-ray examination was performed on the wrists and the bone mineral 
density (BMD) of the wrists was measured to exclude skeletal defects, dislocations, lesions and tumors. An unsealed cuboid container (stainless steel materials) with dimensions of $80 \mathrm{~mm} \times 80 \mathrm{~mm} \times 100 \mathrm{~mm}$ was designed to display the wrists. PALACOS bone cements with low viscosity and $40 \mathrm{~g} \times 20$ boxes (Heraus, Germany) were used to implant the wrists vertically into the cuboid containers by means of the filling technology of bone cement [13], and the bone cements were embedded and fixed until the wrists were completely firm without any movement. Furthermore, the wrists were equipped with a stress sensing system device (Taizhou, China), a microelectronic universal testing machine (Jinan, China) and four static strain test units (Jinan, China) that were connected by wires. The four static strain test units were labeled $A, B, C$ and $D$ units, of which unit $A$ represented the stress of the radioulnar distal palmar unit; unit $B$ represented the stress of the radioulnar distal dorsal unit; unit $C$ represented the stress of the radioulnar proximal palmar unit; and unit $\mathrm{D}$ represented the stress of the radioulnar proximal dorsal unit. Ethical approval was obtained from the Institutional Review Board (Medical Ethics Committee) of the Jiangxi Provincial People's Hospital (Nanchang, China).

Finite element analysis. A healthy male volunteer $(30 \mathrm{y}, 175 \mathrm{~cm}, 65 \mathrm{~kg})$ was screened whose age and forearm size met the requirements of the above cadaver specimens. In addition, to ensure the normal anatomy of the wrist, an X-ray of his wrist was taken to exclude fractures, lesions and other conditions.

\section{Analytical instrumentation}

Biomechanical testing. The digital X-ray camera (Toshiba Electronics Corporation, Tokyo, Japan) and bone mineral density measuring instrument (Cooper surgical Corporation, San Francisco, USA) were supported by Jiangxi Provincial People's Hospital.

Finite element analysis. Analysis was conducted using a dual-source CT (Siemens, Berlin, Germany), a computer, Mimics 19.0 software (Materialise, Leuven, Belgium), Geomagic Studio 12.0 software (Raindrop, North Carolina, USA) and finite element software Abaqus 6.51 (Dassault Simulia Corporation, Providence, USA).

Biomechanical testing methods. The biomechanical experiment in this study was an impact test of static loading within the yield strength. The specimens wearing wrist protectors were divided into experimental groups, and the naked wrist specimens were divided into control groups. In the experimental groups, the wrist protectors were made of $20 \mathrm{~mm}$ thick soft sponge materials and $2 \mathrm{~mm}$ thick polypropylene hard materials. Then, the mechanical axial compression mode was selected, and the strain sensing system was installed on the wrist specimens and connected to static resistance strain gauges. With the palm facing upward, the wrist specimens were fixed according to the above method, 90 degrees perpendicular to the ground, and the pressure hammer was aimed at the center of the navicular and lunate bones. The initial loading speed was set at $2 \mathrm{~mm} / \mathrm{min}$, and the loading range was 0-600 N (Fig. 1). The strain values of all the target units were recorded for every $20 \mathrm{~N}$ load, and the strain values were collected in a table. Each specimen was tested three times under the same conditions, and the strain values of all specimens were obtained and eventually summarized and averaged. 
Three-dimensional (3D) reconstruction of wrist finite element models. The volunteer was scanned transversely by $\mathrm{CT}$, ranging from the proximal forearm to the fingertip. During the scanning, the volunteer referred to the direction of axial loading, and the posture was maintained when the wrist specimens were tested. The established wrist model was set to a wrist dorsiflexion of 75 degrees and a wrist pronation of 10 degrees, and the scanned data were saved in the DICOM format. Then, the data were input into the Mimics19.0 software to initially establish the three-dimensional (3D) geometric model of the wrist. The contours of the forearm cortex, loose tissue and surrounding soft tissue were extracted by the Thresholding and Manual drawing tools, and the STL format data were obtained. Then, the data were input into the Geomagic Studio 12.0 software, and a deeper level of hole filling and smoothing were conducted for each part of the model to prevent the occurrence of poor grids. Finally, the data of the generated solid three-dimensional (3D) model were imported into the Abaqus 6.51 software for statistical analysis. Both cortical bone and cancellous bone were simulated by linear elastic material, and soft tissue was simulated by Mooney-Rivlin hyperelastic material. Previous literature was referenced to set the material properties (Table 2). [14]

Statistical analysis. The data obtained above were statistically analyzed by SPSS 22.0 software (SPSS, Chicago, USA) with the paired t-test. $P<0.05$ was regarded as a difference with statistical significance.

\section{Results}

The experimental results for the control group. In the axial-loading simulated experiment of the control group, the stress magnitudes and properties of the wrist model were different. Among them, the stresses of palmar units were apparently higher than the stresses of dorsal units, and the stresses of palmar units mainly took the form of pressure. The initial stage of the dorsal units took the form of pressure, but the whole process was dominated by tension. The equivalent stresses of the radial distal and proximal palmar units under $600 \mathrm{~N}$ load were $36.6 \mathrm{MPa}$ and $58.5 \mathrm{MPa}$, respectively. Under the same loading conditions, the equivalent stresses of the ulnar distal and proximal palmar units were close to those on the radial distal and proximal palmar units at $37.9 \mathrm{MPa}$ and $37.4 \mathrm{MPa}$, respectively. In all the groups of units, the stresses of the dorsal units were lower than those of the palmar units. Moreover, the stresses of the ulnar distal and proximal dorsal units and radioulnar proximal dorsal units took the form of pressure in the initial stage of the experiment and transformed into tension in the middle and late stages. The maximum stresses of the radioulnar distal dorsal units were 9.3 $\mathrm{MPa}$ and $13.4 \mathrm{MPa}$, while the maximum stresses of the radioulnar proximal dorsal units were $10.5 \mathrm{MPa}$ and $15.6 \mathrm{MPa}$.

The experimental results for the experimental group. In the axial-loading simulated experiment of the experimental group, the stress magnitude and properties of the wrist model were also different. The stresses of the palmar units were apparently higher than the stresses of the dorsal units, and every unit was mainly dominated by pressure. The initial stage of the radial proximal dorsal unit took the form of pressure, which transformed into tension in the middle and late stages. In addition, tension was the main manifestation at the radioulnar distal and dorsal units and ulnar proximal dorsal unit. Under the same loading conditions, the stresses of the radioulnar proximal palmar units were all higher than those of the 
radioulnar distal palmar units. The equivalent stresses of the radioulnar proximal palmar units under 600 $\mathrm{N}$ load were $36.6 \mathrm{MPa}$ and $58.5 \mathrm{MPa}$, while the equivalent stresses of the radioulnar distal palmar units were 20.2 MPa and 24.2 MPa. In all the groups of units, the stresses of the dorsal units were lower than those of the palmar units. The maximum stresses on the radioulnar distal dorsal units were $5.6 \mathrm{MPa}$ and 4.7 $\mathrm{MPa}$, while the maximum stresses on the radioulnar proximal dorsal units were $10.2 \mathrm{MPa}$ and 13.2 $\mathrm{MPa}$, respectively (Fig. 2).

Comparison between the experimental results of the control group and the experimental group. A comparison between the stress peaks and declines of the two groups was derived as shown in Table I. In all 8 groups, except for the radioulnar proximal and dorsal units, there were no significant stress differences between the control group and the experimental group in the late stage of the experiment. However, the stresses of the remaining 6 units in the experimental group decreased by $44 \%$ compared with the control group on average (Fig. 3).

The establishment of the three-dimensional (3D) finite element model of the wrist. Based on the extension, flexion, retraction and rotation of a normal human wrist, the three-dimensional (3D) finite element model of the wrist was composed of 136,897 units of bone, 9166 units of cartilage and 228,893 units of soft tissue, totaling 374,956 units (Table 3 ).

The stress distribution in the models of the control group and the experimental group. In the late stage of the experiments, the stress distributions in the models of the control group and the experimental group are shown in Fig. 4. The results of the finite element analysis confirmed the conclusions of the biomechanical experiments described above. Except for the radioulnar proximal and dorsal units, there were no significant stress color differences between the control group and the experimental group at the end of the experiments. However, the stress colors of the remaining 6 units in the experimental group were lighter than those in the control group, indicating that the stresses of the experimental group were lower than those of the control group. This result further verified the scientificity and effectiveness of the biomechanical experiments above.

\section{Discussion}

As the aging process of the population continues to evolve, the increase in the proportion of elderly people has led to a surge in patients with wrist fractures to a certain extent [15-17]. Especially in middleaged and elderly women due to menopause and estrogen loss, physical function and the ability to deal with emergencies has inevitably declined, which results in a high incidence of wrist fractures $[13,18-20]$. In addition, wrist fractures are common in young and middle-aged people during daily exercise and work $[21,22]$. To improve the ability to prevent wrist fractures at different ages and update wrist protectors with poor effectiveness in the current state, this study combined a mechanical experiment on cadaveric wrists with finite element wrist simulation analysis to evaluate the effects of external forces on the wrist from multiple angles. 
During the axial-loading experiments, we found that despite the complex anatomical structure of the wrist joint, the external forces were mainly transmitted from the navicular and lunate bones down to the radioulnar joints and then continued to the proximal end of the forearm. In this process, the radioulnar joint was regarded as a composite joint, and compressive deformation occurred in the radioulnar palmar units, presenting as compressive stress. The tensile deformation occurred in the radioulnar dorsal units, presenting as tensile stress. In addition, under normal circumstances, during the deformation of the radioulnar joint under external forces, the palmar and dorsal units with the same axis distance had the same bending moments [23]. However, in this experiment, the axial center was biased to the dorsal units under the impact, and all the units had larger palmar bending moments and smaller dorsal bending moments, which explains why the absolute values of the palmar units were greater than those of the stress on the dorsal units.

Second, a comparison of the data from the two groups showed that wearing a wrist protector can effectively reduce the stress on the radioulnar distal palmar, radioulnar proximal palmar and radioulnar distal dorsal units but has no obvious influence on the radioulnar proximal dorsal units. In the experimental group, the stresses of the radioulnar distal palmar and dorsal units were apparently reduced by $44 \%$ compared with the control group on average, which was related to the absorption and shunting of the impact load on the wrist protector. Therefore, when designing and improving a wrist protector, it is reasonable to place the stress center on the radioulnar distal palmar and dorsal units. Similar to the conclusion of this experiment, Sun et al. [24] designed a hip protector and screened 3 volunteers to perform simulated human side fall tests at a certain intensity. The results indicated that the average peak impact force could reach $(1738.88 \pm 215.66) \mathrm{N}$ in the group without hip pads, while the average peak impact force in the group with hip pads apparently increased to $(1907.44 \pm 441.42) \mathrm{N}$. This result reflected that wearing a hip protector can increase the peak impact force on the hip, preventing the occurrence of hip fracture to a certain extent.

In addition, there are certain limitations in this study. First, cadaveric specimens lack the soft tissue tension and stress protection mechanisms of the normal human body and cannot accurately reflect the true stress and strain on the normal human wrist [25-27]. Second, the experimental sample size is only 6 cases, and the sample size is relatively small. Third, the force mechanism of wrist fracture caused by external force impact is complex. This experiment is limited to the vertical axial loading of the wrist joint, which simplifies the actual forces on the human body. Finally, the finite element analysis itself also has certain limitations, that is, the simulation can only approximate the real situation, and the authenticity and validity of the results need to be mutually verified with experiments, which leads to deviation from experimental results to a certain extent [28].

\section{Conclusions}

In conclusion, this study combined a mechanical experiment on cadaveric wrists with finite element wrist simulation analysis. It can be concluded that the stresses on the radioulnar palmar units were high and took the form of pressure, while the stresses on the radioulnar dorsal units were relatively lower and took 
the form of tension. Within the range of physiological loads, wearing a wrist protector can significantly reduce the stresses on the radioulnar distal palmar, radioulnar proximal palmar and radioulnar distal dorsal units but has no obvious influence on the radioulnar proximal dorsal units. These results provide a theoretical basis for simulation experiments on wrist fracture and the optimal design of wrist protectors.

\section{Abbreviations}

BMD

bone mineral density

\section{Declarations}

\section{Ethics approval and consent to participate}

Ethical approval was obtained from the Institutional Review Board (Medical Ethics Committee) of the Jiangxi Provincial People's Hospital (Nanchang, China).

\section{Consent for publication}

Not applicable

\section{Availability of data and materials}

The datasets used and analyzed during the current study are available from the corresponding author on reasonable request.

\section{Competing interests}

The authors declare that they have no competing interests

\section{Funding}

This study was supported by grants from the National Natural Science Foundation of China (Grant No. 81460346) and General Technology Plan of Jiangxi Provincial Health Planning Commission (No. 20181008).

\section{Authors' contributions}

JYH designed the study and wrote the manuscript. LZ collected and processed the data. All authors read and approved the final manuscript.

\section{Acknowledgements}

None. 


\section{References}

1. de Putter CE, Selles RW, Polinder S, Hartholt KA, Looman CW, Panneman MJ, Verhaar JA, Hovius SE, van Beeck EF: Epidemiology and health-care utilisation of wrist fractures in older adults in The Netherlands, 1997-2009. Injury 2013, 44(4):421-426.

2. Gregson CL, Carson C, Amuzu A, Ebrahim S: The association between graded physical activity in postmenopausal British women, and the prevalence and incidence of hip and wrist fractures. Age and ageing 2010, 39(5):565-574.

3. Jung HY, Chang M, Kim KM, Yoo W, Jeon BJ, Hwang GC: Effect of Wrist Joint Restriction on Forearm and Shoulder Movement during Upper Extremity Functional Activities. Journal of physical therapy science 2013, 25(11):1411-1414.

4. Park K, Chang PH, Kang SH: In Vivo Estimation of Human Forearm and Wrist Dynamic Properties. IEEE transactions on neural systems and rehabilitation engineering : a publication of the IEEE Engineering in Medicine and Biology Society 2017, 25(5):436-446.

5. Erhart S, Lutz M, Arora R, Schmoelz W: Measurement of intraarticular wrist joint biomechanics with a force controlled system. Medical engineering \& physics 2012, 34(7):900-905.

6. Schreck MJ, Kelly M, Canham CD, Elfar JC: Techniques of Force and Pressure Measurement in the Small Joints of the Wrist. Hand (New York, NY) 2018, 13(1):23-32.

7. Kramer A, Allon R, Wolf A, Kalaiman T, Lavi I, Wollstein R: Anatomical Wrist Patterns on Plain Radiographs. Current rheumatology reviews 2017.

8. Crandall CJ, Hovey KM, Cauley JA, Andrews CA, Curtis JR, Wactawski-Wende J, Wright NC, Li W, LeBoff MS: Wrist Fracture and Risk of Subsequent Fracture: Findings from the Women's Health Initiative Study. Journal of bone and mineral research : the official journal of the American Society for Bone and Mineral Research 2015, 30(11):2086-2095.

9. Gallagher JC: Advances in osteoporosis from 1970 to 2018. Menopause (New York, NY) 2018, 25(12):1403-1417.

10. Gong H, Zhang M, Fan Y, Kwok WL, Leung PC: Relationships between femoral strength evaluated by nonlinear finite element analysis and BMD, material distribution and geometric morphology. Annals of biomedical engineering 2012, 40(7):1575-1585.

11. Palacios S, de Villiers TJ, Nardone Fde C, Levine AB, Williams R, Hines T, Mirkin S, Chines AA: Assessment of the safety of long-term bazedoxifene treatment on the reproductive tract in postmenopausal women with osteoporosis: results of a 7-year, randomized, placebo-controlled, phase 3 study. Maturitas 2013, 76(1):81-87.

12. Palacios S, Silverman SL, de Villiers TJ, Levine AB, Goemaere S, Brown JP, De Cicco Nardone F, Williams R, Hines TL, Mirkin S et al: A 7-year randomized, placebo-controlled trial assessing the longterm efficacy and safety of bazedoxifene in postmenopausal women with osteoporosis: effects on bone density and fracture. Menopause (New York, NY) 2015, 22(8):806-813. 
13. Belaid D, Vendeuvre T, Bouchoucha A, Bremand F, Breque C, Rigoard P, Germaneau A: Utility of cement injection to stabilize split-depression tibial plateau fracture by minimally invasive methods: A finite element analysis. Clinical biomechanics (Bristol, Avon) 2018, 56:27-35.

14. Gislason MK, Nash DH, Nicol A, Kanellopoulos A, Bransby-Zachary M, Hems T, Condon B, Stansfield B: A three-dimensional finite element model of maximal grip loading in the human wrist. Proceedings of the Institution of Mechanical Engineers, Part H: Journal of Engineering in Medicine 2009, 223(7):849-861 .

15. Antoniadou E, Kouzelis A, Diamantakis G, Bavelou A, Panagiotopoulos E: Characteristics and diagnostic workup of the patient at risk to sustain fragility fracture. Injury 2017, 48 Suppl 7:S17-s23.

16. Frontera WR: Physiologic Changes of the Musculoskeletal System with Aging: A Brief Review. Physical medicine and rehabilitation clinics of North America 2017, 28(4):705-711.

17. Walters S, Khan T, Ong T, Sahota O: Fracture liaison services: improving outcomes for patients with osteoporosis. Clinical interventions in aging 2017, 12:117-127.

18. Gallagher JC, Palacios S, Ryan KA, Yu CR, Pan K, Kendler DL, Mirkin S, Komm BS: Effect of conjugated estrogens/bazedoxifene on postmenopausal bone loss: pooled analysis of two randomized trials. Menopause (New York, NY) 2016, 23(10):1083-1091.

19. Khan A, Fortier M: Osteoporosis in menopause. Journal of obstetrics and gynaecology Canada : JOGC = Journal d'obstetrique et gynecologie du Canada : JOGC 2014, 36(9):839-840.

20. Stagraczynski M, Kulczyk T, Leszczynski P, Meczekalski B: [Number of teeth and hormonal profile of postmenopausal women with osteoporosis, osteopenia and normal bone mineral density-a preliminary study]. Polski merkuriusz lekarski : organ Polskiego Towarzystwa Lekarskiego 2015, 39(232):214-218.

21. Beerekamp MSH, de Muinck Keizer RJO, Schep NWL, Ubbink DT, Panneman MJM, Goslings JC: Epidemiology of extremity fractures in the Netherlands. Injury 2017, 48(7):1355-1362.

22. Clement ND, Duckworth AD, Wickramasinghe NR, Court-Brown CM, McQueen MM: Does socioeconomic status influence the epidemiology and outcome of distal radial fractures in adults? European journal of orthopaedic surgery \& traumatology : orthopedie traumatologie 2017, 27(8):1075-1082.

23. Sherman KM, Miller GJ, Wronski TJ, Colahan PT, Brown M, Wilson W: The effect of training on equine metacarpal bone breaking strength. Equine veterinary journal 1995, 27(2):135-139.

24. PD S: Experimental and finite element analysis of the influence of lateral fall height and hip protector on hip impact. Southern Medical University 2012(In Chinese).

25. Hogel F, Mair S, Eberle S, Weninger P, von Oldenburg G, Augat P: Distal radius fracture fixation with volar locking plates and additional bone augmentation in osteoporotic bone: a biomechanical study in a cadaveric model. Archives of orthopaedic and trauma surgery 2013, 133(1):51-57.

26. Kang L, Dy CJ, Wei MT, Hearns KA, Carlson MG: Cadaveric Testing of a Novel Scapholunate Ligament Reconstruction. Journal of wrist surgery 2018, 7(2):141-147. 
27. Mirarchi AJ, Hoyen HA, Knutson J, Lewis S: Cadaveric biomechanical analysis of the distal radioulnar joint: influence of wrist isolation on accurate measurement and the effect of ulnar styloid fracture on stability. The Journal of hand surgery 2008, 33(5):683-690.

28. Sannino G, Barlattani A: Mechanical evaluation of an implant-abutment self-locking taper connection: finite element analysis and experimental tests. The International journal of oral \& maxillofacial implants 2013, 28(1):e17-26.

\section{Tables}

Table 1. The comparison of stress peak and decline between two groups

\begin{tabular}{cccc}
\hline & The peak of control group/MPa & The peak of experimental group/MPa & The average decline/MPa \\
\hline Radial distal palmar unit & 36.6 & 20.2 & $44.8 \%$ \\
Ulnar distal palmar unit & 37.9 & 24.3 & $35.9 \%$ \\
Radial distal dorsal unit & 9.4 & 5.7 & $39.4 \%$ \\
Ulnar distal dorsal unit & 13.4 & 4.8 & $64.1 \%$ \\
Radial proximal palmar unit & 58.5 & 30.1 & $48.5 \%$ \\
Ulnar proximal palmar unit & 37.4 & 24.9 & $33.4 \%$ \\
Radial proximal dorsal unit & 10.5 & 10.3 & $1.9 \%$ \\
Ulnar proximal dorsal unit & 15.3 & 13.8 & $6.7 \%$ \\
\hline
\end{tabular}

Table 2. Material properties used in finite-element model

\begin{tabular}{cccc}
\hline & Density $\left(\mathrm{g} / \mathrm{cm}^{3}\right)$ & Young's Modulus (E: MPa) & Poisson's Ratio $(\mu)$ \\
& & & \\
\hline Bone & 1.4 & 18.570 & 0.20 \\
Cancellous bone & 1.0 & 3.635 & 0.42 \\
Soft tissue & 0.749 & $\mathrm{C} 10=0.4, \mathrm{C} 01=0.1, \mathrm{D} 1=0.3$ & - \\
Soft brace & 1.4 & 10 & 0.3 \\
Rigid brace & 1.4 & 1500 & 0.3 \\
\hline
\end{tabular}

Table 3. The number of nodes, unit types and numbers of the finite element model

\begin{tabular}{ccccccc}
\hline & Bone & Cancellous bone & Soft tissue & Rigid ground & Soft brace & Rigid brace \\
\hline Unit number & 136897 & 9166 & 228893 & 2500 & 12048 & 3984 \\
Nodes & 228808 & 20499 & 356621 & 2603 & 4196 & 2090 \\
Unit Type & C3D10M & C3D10M & C3D10M & S4R & C3D10M & S3R \\
\hline
\end{tabular}

\section{Figures}




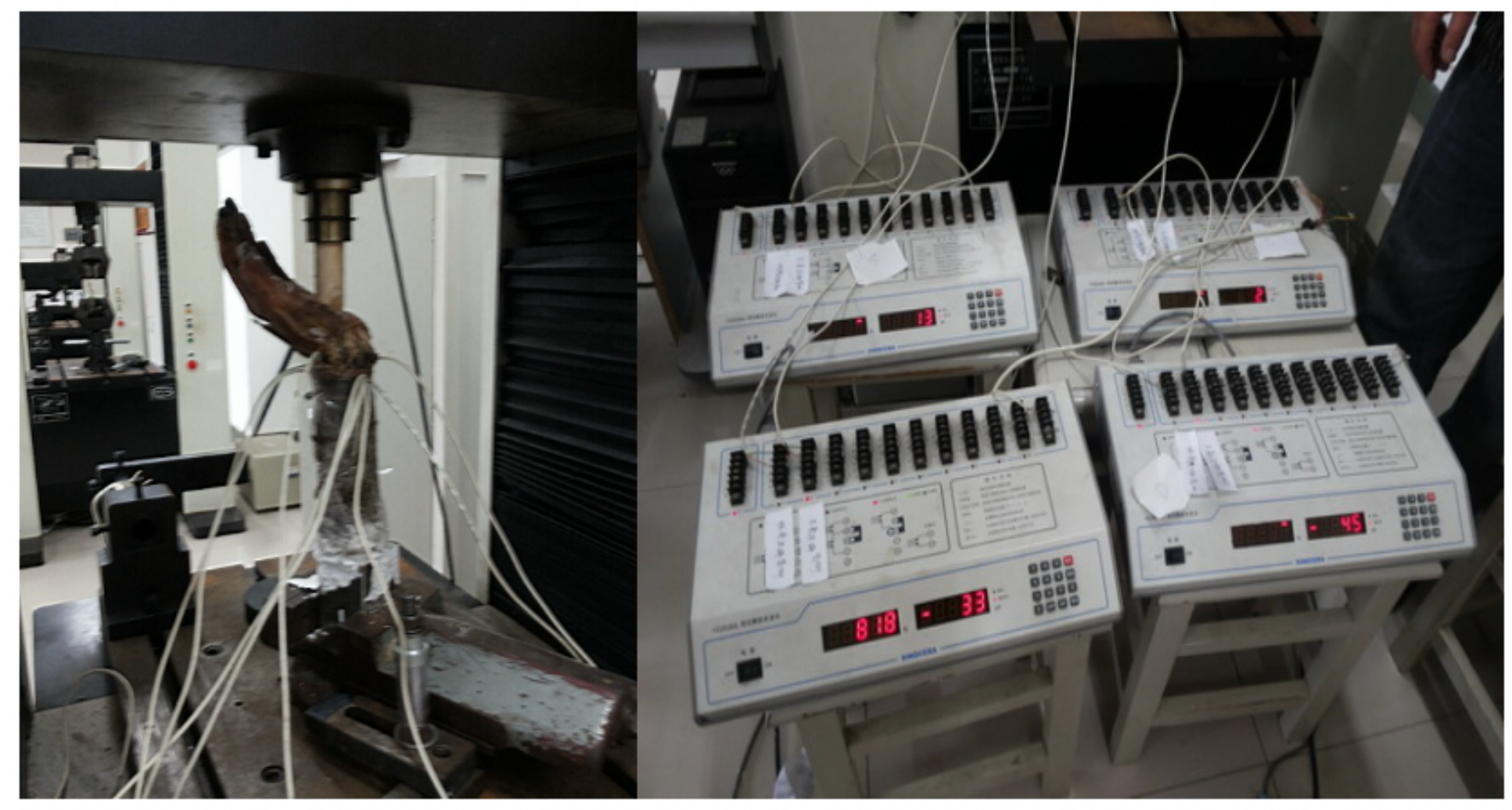

Figure 1

(A) Axial-loading experiment on wrist specimens. (B) Establishment of four static strain test units.
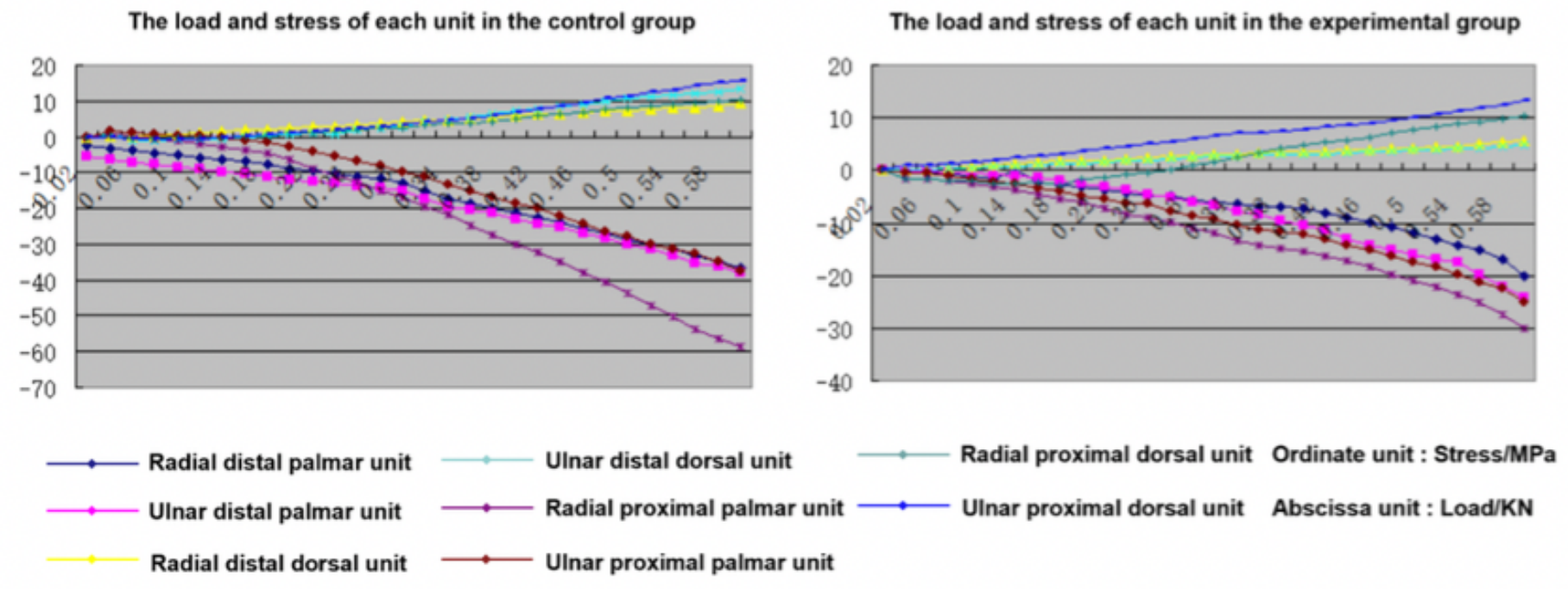

Figure 2

Comparison of the load and stress of each unit in the control group and the experimental group. 

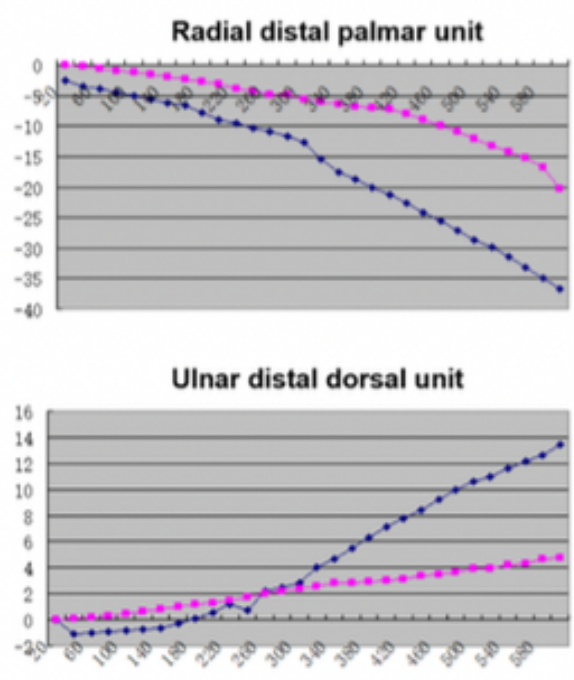

Radial proximal dorsal unit

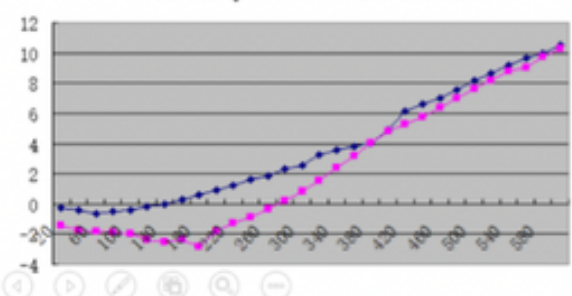

Ulnar distal palmar unit

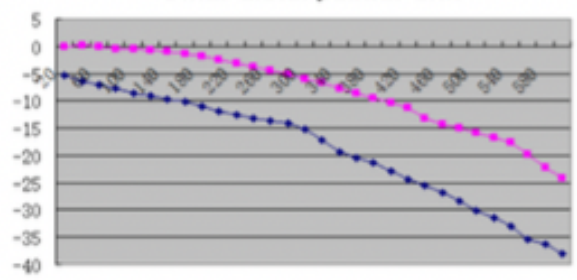

Radial proximal palmar unit

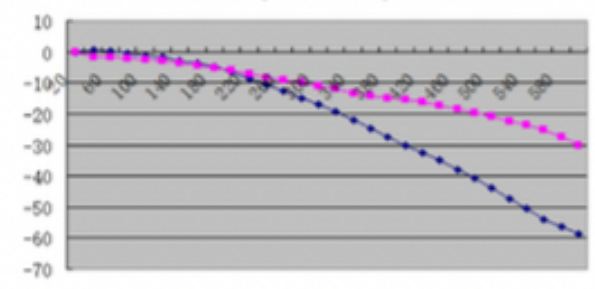

Ulnar proximal dorsal unit

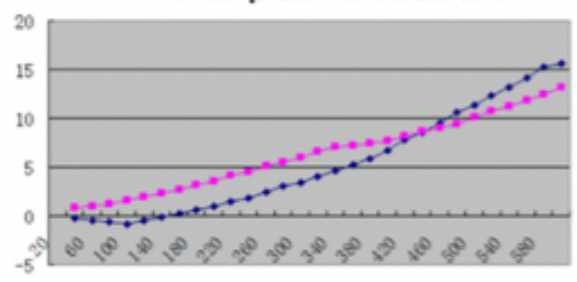

Radial distal dorsal unit

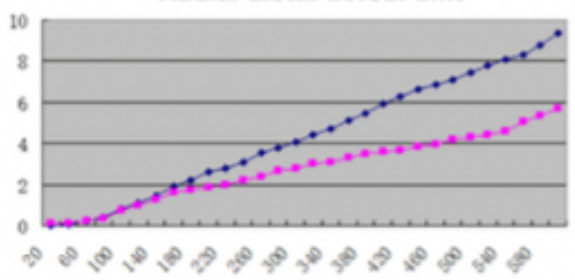

Ulnar proximal palmar unit

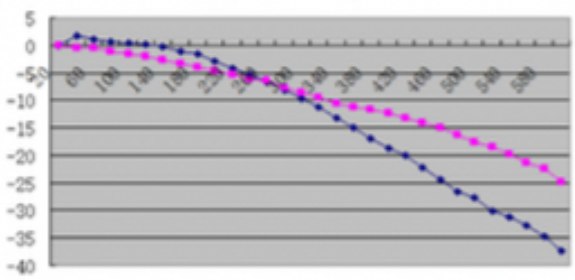

The control group

The experimental group

Ordinate unit : Stress/MPa

Abscissa unit : Load/N

\section{Figure 3}

The load and stress curves of each unit for the control group and the experimental group.

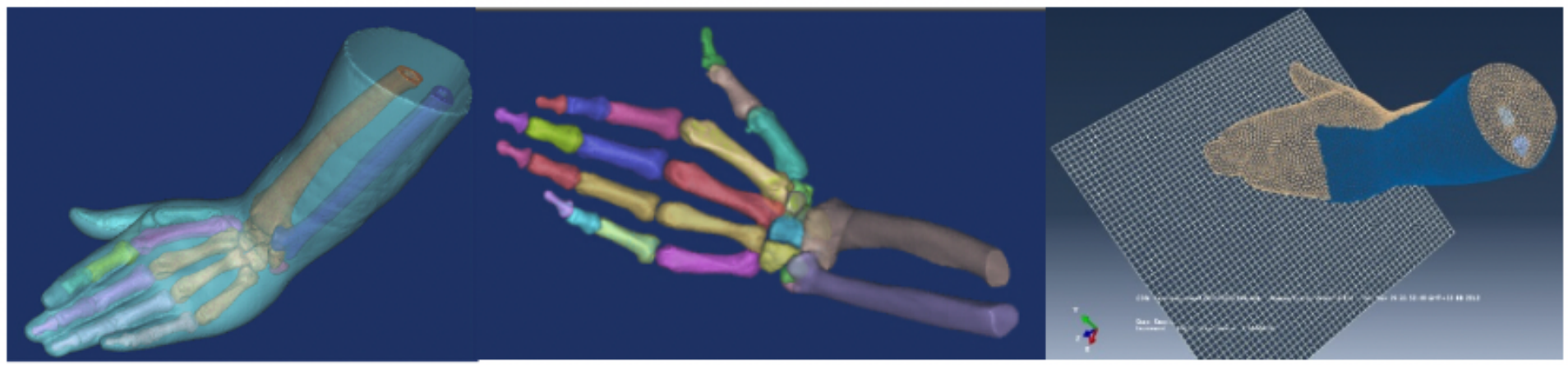

\section{Figure 4}

The models of wrist with $(A)$ or without the wrist protector $(B)$ 


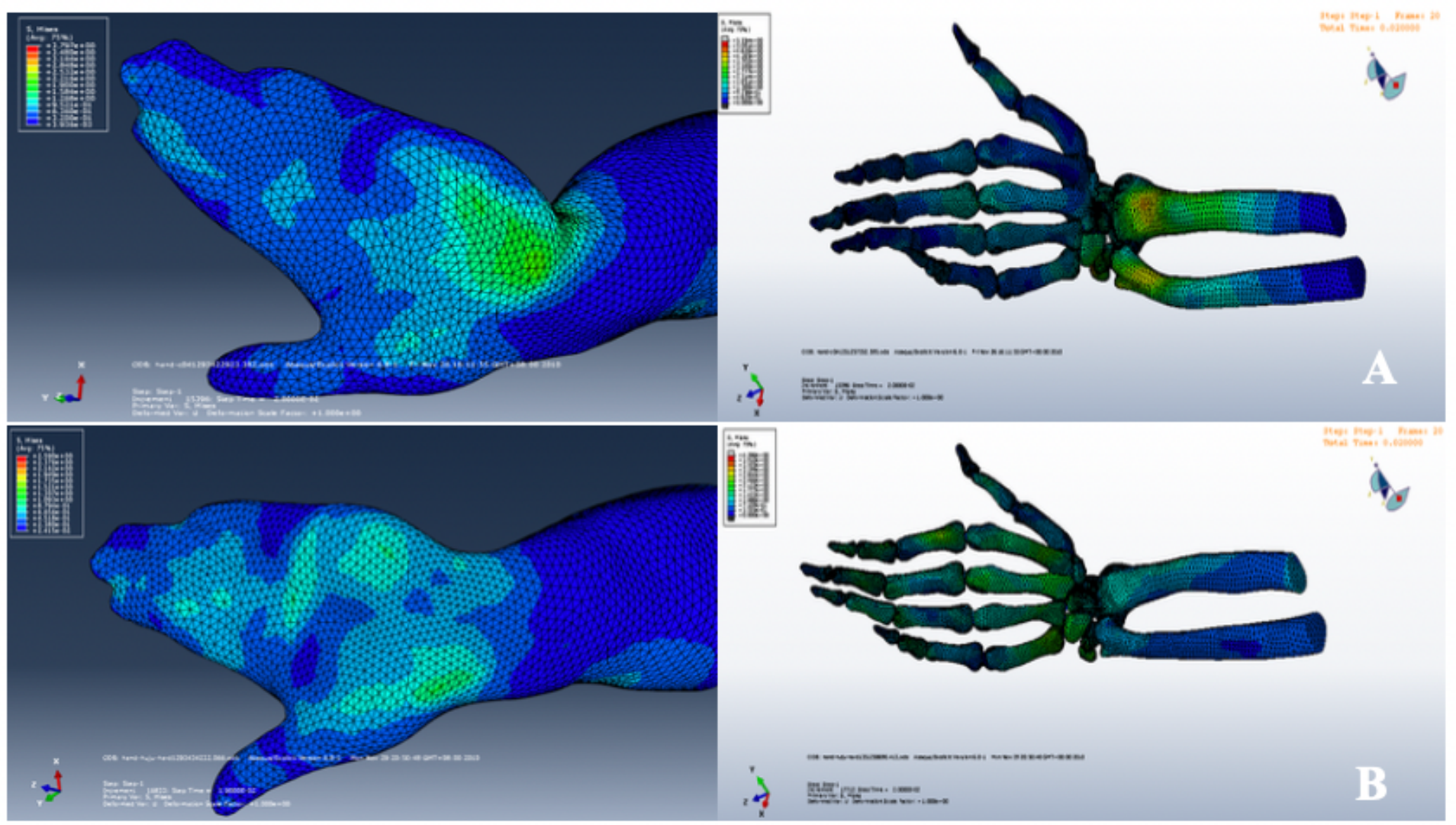

\section{Figure 5}

The stress distributions in the finite analysis models of the control group and the experimental group. (A) Without the buffer of the wrist protector, the stress color of the control group was apparently deeper than that of the experimental group in the radioulnar distal palmar, radioulnar proximal palmar and radioulnar distal dorsal units. (B) Under the protection of the wrist protector, the overall stress color of the experimental group was light. 